\title{
Identification of Children With Prenatal Alcohol Exposure
}

\author{
G. Coriale • D. Fiorentino • P. W. Kodituwakku • \\ L. Tarani • G. Parlapiano • B. Scalese • M. Ceccanti
}

Published online: 24 April 2014

(C) Springer International Publishing Switzerland 2014

\begin{abstract}
The term "fetal alcohol spectrum disorders" (FASD) denotes the broad spectrum of morphological changes and functional deficits seen in children exposed to alcohol prenatally. While some children on the spectrum show the characteristic pattern of malformations called "fetal alcohol syndrome" (FAS), a significant proportion of alcohol-exposed children do not evidence clinically identifiable morphological alterations. The term "alcohol-related neurodevelopmental disorder" (ARND) is used to label the latter group. The identification of children with ARND has proven to be challenging because of the lack of clinically discernable physical
\end{abstract}

\author{
G. Coriale $(\bowtie) \cdot$ D. Fiorentino $\cdot$ B. Scalese \\ Lazio Regional Center for Alcohol (CRARL), V.le dell'Università \\ 37, 00185 Rome, Italy \\ e-mail: gcoriale@tin.it \\ D. Fiorentino \\ e-mail: d.fiorentino@libero.it \\ B. Scalese \\ e-mail: brunascalese@libero.it \\ P. W. Kodituwakku \\ Center for Development and Disability, University of New Mexico \\ School of Medicine, 2300 Menaul Blvd. NE, Albuquerque, \\ NM 87107, USA \\ e-mail: pkodituwakku@salud.unm.edu \\ L. Tarani · G. Parlapiano \\ Department of Pediatrics and Pediatric Neuropsychiatry, Sapienza \\ University of Rome, V.le Regina Elena, 324, 00161 Rome, Italy \\ L. Tarani \\ e-mail: luigi.tarani@uniroma1.it \\ G. Parlapiano \\ e-mail: parlapiano.giovanni@gmail.com

\section{Ceccanti} \\ Department of Clinical Medicine, Sapienza University of Rome, \\ Viale del Policlinico, 00161 Rome, Italy \\ e-mail: mauro.ceccanti@uniroma1.it
}

signs. Therefore, some investigators have used the strategy of assessing maternal drinking during pregnancy and then tracking developmental outcomes in offspring longitudinally. Other methods that investigators have utilized to identify children with prenatal alcohol exposure in population-based studies include multi-source surveillance and active case ascertainment. In the current review, we discuss the merits and demerits of these methodologies and then present novel methods of identifying prenatal alcohol exposure (e.g., biomarkers) and subtle effects of morphological alterations and neural effects (e.g., neuroimaging).

Keywords FASD · Fetal alcohol syndrome spectrum disorders - Alcohol related neurodevelopmental disorder . ARND · FAS · Fetal alcohol syndrome · Surveillance · Neuroimaging $\cdot$ Biomarkers $\cdot 3 \mathrm{D}$ facial photography

\section{Introduction}

Considerable attention has been devoted to fetal alcohol spectrum disorders since the deleterious effects of prenatal alcohol exposure (PAE) on the developing fetus were first reported in the medical literature over 40 years ago $[1,2]$. As described in other papers in this special edition, the term "fetal alcohol spectrum disorders" (FASD) refers to the entire range of physiological, behavioral, and neurocognitive abnormalities observed in the offspring of mothers who drank during pregnancy [3]. At one end of this range is a set of malformations, which Jones et al. [2] labeled "fetal alcohol syndrome" (FAS). FAS is characterized by a characteristic pattern of minor anomalies on the face (small palpebral fissures, a smooth philtrum, and a thin vermillion border), growth restrictions, and central nervous system dysfunction, as indexed by morphological differences (e.g., microcephaly) and behavioral problems (e.g., hyperactivity) [2]. It is now known that most 
alcohol-exposed children show these malformations to varying degrees, with a substantial proportion showing only cognitive and behavioral problems. The Institute of Medicine report on fetal alcohol syndrome [4] introduced the term "alcohol-related neurodevelopmental disorder" (ARND) to describe the group that exhibits cognitive and behavioral problems in the absence of clinically identifiable morphological anomalies. The Identification of children with ARND in clinical practice has proven to be a challenging problem because the clinical presentation of these children strongly resembles that of other neurodevelopmental disorders. It has been well documented that children with FASD show a range of co-morbid conditions, including attention deficit-hyperactivity disorder (ADHD) and depression [5]. Despite recent attempts to define a behavioral phenotype of FASD [6], there is no signature behavioral or cognitive profile uniquely associated with prenatal alcohol exposure (see Kodituwakku and Kodituwakku in the current volume). In the absence of clinically identifiable physical signs and a signature cognitivebehavioral profile, it is imperative that the prenatal alcohol exposure be established for making an alcohol-related diagnosis. However, it has proven to be difficult to obtain accurate information on prenatal alcohol exposure, because of the underreporting of drinking or unavailability of birth mothers for interviews. In view of the foregoing difficulties in the diagnosis of FASD, researchers have developed different methodologies to identify children with prenatal alcohol exposure. As shown in Fig. 1, some investigators have sought to identify children with FASD through the assessment of prenatal substance abuse by "at-risk" mothers. This methodology has been employed in large-scale longitudinal studies conducted in North America and Europe. The strategy utilized in these studies has involved the systematic collection of detailed information on drug and alcohol use during pregnancy through maternal and informant interviews and then tracking of the development of the offspring from birth to adulthood [7]. Since maternal self-reports of alcohol use can be unreliable, some researchers have suggested the importance of collecting biomarkers (e.g., meconium) to verify prenatal exposure to alcohol [8-10]. Another approach has focused on directly assessing the child to obtain evidence of prenatal alcohol exposure. For example, epidemiological studies conducted in the USA, South Africa, and Italy have screened community-based samples, using morphological and behavioral criteria to identify potential cases of prenatal alcohol exposure $[11,12 \bullet, 13 \bullet]$. Researchers have recently utilized neuroimaging methods $[14,15]$ and three-dimensional (3D) facial photography [16] to detect subtle markers of prenatal alcohol exposure [17].

As shown in Fig. 1, large-scale longitudinal studies have obtained exposure data during pregnancy and then child data (morphology and cognitive functioning) at different stages of development. The main purpose of these studies has been to evaluate the developmental outcomes of prenatal alcohol exposure rather than to estimate prevalence rates of FASD. However, detailed data gathered through maternal interviews, cognitive assessments, and morphological evaluations permit estimation of the rate of FASD among those who are exposed [18]. In contrast, the express purpose of the active case ascertainment methodology has been to estimate prevalence rates and risk factors rather than the developmental outcomes of exposure. This methodology therefore entails collecting retrospective data on maternal drinking during pregnancy, as well as morphological and cognitive data on children. The Centers for Disease Control and Prevention (CDC) in the USA have employed a standardized methodology for the surveillance of fetal alcohol syndrome, in which investigators have estimated the prevalence of FAS through review of records from multiple sources [19]. In this paper, we briefly review the merits and demerits of the above methodologies and then discuss recent methodological advances in the identification of prenatal alcohol exposure and the morphological and functional sequelae of alcohol-induced damage. First, we present the surveillance methodology employed by the CDC, followed by a discussion of the clinic-based studies. Then, we discuss the active case ascertainment methodology that has been used in the USA and other countries. In the final section, we focus on the emerging methodologies to determine prenatal alcohol exposure (e.g., biomarkers) and to assess morphological sequelae (3D photography) and functional sequelae (e.g., neuroimaging).

\section{Surveillance of Fetal Alcohol Syndrome}

The Fetal Alcohol Surveillance Network (FASSNet), supported by the CDC, exemplifies the use of surveillance methodology to identify children with FAS. This study involved five states in the USA — namely, Alaska, Arizona, Colorado, New York, and Wisconsin - and employed a "standardized, multisource" approach [20]. At all sites, a standardized case definition of confirmed or probable FAS was developed, which incorporated the primary diagnostic criteria for FAS. These included abnormal facial features consistent with FAS or two cardinal facial anomalies (short palpebral fissures, an abnormal philtrum, a thin upper lip); central nervous system dysfunction (e.g., microcephaly, IQ $\leq 1$ standard deviation from the mean, etc.); and growth restrictions (intrauterine weight or height corrected for gestational age $\leq 10$ th percentile; postnatal height or weight $\leq 10$ th percentile). The investigators tapped into multiple sources of data (e.g., hospitals, birth defect monitoring programs, genetic clinics, developmental clinics, early intervention programs, and Medicaid files) to identify cases. A common electronic data extraction form was used, and quality assurance procedures were instituted [20] for fidelity checks. Initial analyses of the data from children born during 1995-1997 showed that the rates of confirmed or 


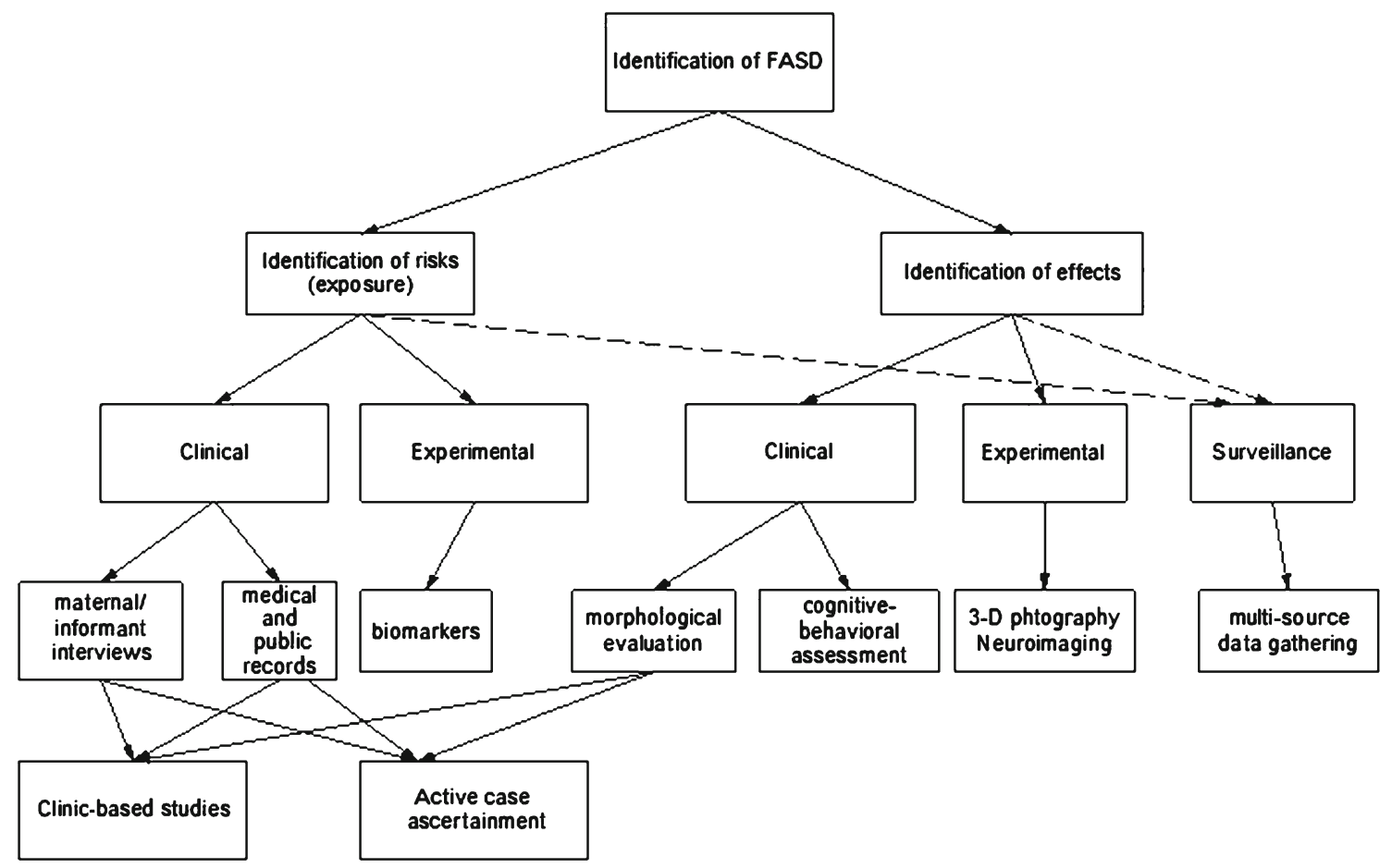

Fig. 1 Methodologies used to identify children with fetal alcohol spectrum disorders (FASD). The dashed lines indicate that the information on exposure and effects are gathered through record reviews

probable FASD varied from 0.4 to 1.5 per 1,000 live births across the five states, compared with rates of 0.2 per 1,000 of all births from 1979 to 1992 [21], 0.37 per 1,000 in 1992, and 0.67 per 1,000 in 1993 [22]. Such a method can be defined as passive, because it is based on counting, ex post, how many cases of the indexed pathology occurred during a specific time period. More active methods combine data coming from different sources, as a case can be registered in different records [23]. As an example, a study performed in Alaska, using different sources (from hospital admissions, pediatricians, birth certificates, and programs for genetic diseases, disability, or education) identified a FAS prevalence rate of $0.2-0.3$ per 1,000 in the non-Native population [24]. Data from 15 such studies conducted in the USA estimated a mean FAS prevalence rate of 0.85 per 1,000 live births (median 0.27 ). The main advantage of the surveillance method concerns its cost effectiveness. For example, the CDC-funded FASSNet project collected population-based data on FAS from multiple source record-based methods from a number of states in the USA at a low cost. A major limitation of the record-based method was its failure to identify cases on the spectrum that did not meet the criteria of fetal alcohol syndrome. It has been estimated that the proportion of children on the spectrum without dysmorphia may be three times as high as that of FAS [18]. This method may also underestimate the rates of FAS because FAS and other genetic disorders are not often diagnosed at birth $[11,25]$. Furthermore, most health centers may not have clinicians to diagnose fetal alcohol syndrome.

\section{Clinic-Based Longitudinal Studies}

As mentioned above, clinic-based studies involve following pregnant women during pregnancy, collecting data on their alcohol consumption, and then tracking the developmental trajectories of alcohol-exposed infants after they are born. Having reviewed this type of study, Abel and Sokol [26-28] found higher prevalence rates of FAS in the USA in comparison with European countries, although the reported amounts of maternal drinking were higher in the latter countries. These investigators dubbed this the "American paradox" to describe this discrepancy between prevalence rates and reported alcohol consumption [29, 30]. Abel's 1995 review [28] described 35 studies, including studies from Europe and Australia. The studies from Europe and Australia, which involved predominantly Caucasian middle class populations, reported low rates of FASD and no cases of FAS. In this review, Abel [28] estimated relatively lower rates of FASD in other western countries $(0.97$ per 1,000$)$ than in the USA $(1.95$ per 1,000$)$. May et al. [31] suggested that the paradox could be explained by the fact that in the USA, more binge drinkers exist than in Europe, where people drink more on a daily basis. In fact, binge drinking is known to increase the blood alcohol level sharply, which is a known risk factor for FAS [29]. Several 
studies have documented that binge drinking is correlated with morphological changes and cognitive deficits in exposed children $[32,33]$. Another explanation for the paradox lies in sample differences in socioeconomic status (SES). Abel [28] demonstrated that the prevalence of FAS is higher among lower SES groups $(2.29$ per 1,000$)$ than in higher SES groups $(0.26$ per 1,000). The moderating effect of SES on FAS is further illustrated by recent studies of neurobehavioral functioning in children exposed to light drinking during pregnancy. Data from two large-scale population-based cohorts from the UK (the Millennium Cohort and Devon Cohort) indicated that light drinking during pregnancy does not pose a risk for cognitive-behavioral problems in exposed children [34-36]. Included in these cohorts were predominantly white, middle class families. In contrast, Sood et al. [37] observed a doseresponse effect of alcohol exposure on behavior in children with low levels of exposure. Enrolled in the latter study were African American women, predominantly from lower socioeconomic backgrounds. The merits of longitudinal studies of FASD include their ability to delineate the factors that lead to ARND among alcohol-exposed children. One of the first reports on the prevalence of FASD came from a clinic-based longitudinal study - namely, the Seattle 500 study [18]. Sampson et al. [18] estimated the prevalence of FASD to be 9.1 per 1,000 live births. Since developmental risk factors, including alcohol exposure, are identified during pregnancy, this design also allows initiation of early interventions. A fundamental weakness in clinic-based cohorts, particularly from North America, concerns a self-selection bias. For example, the Detroit, Atlanta, and Pittsburgh cohorts included predominantly African American women from low socioeconomic backgrounds who attended university-based clinics.

\section{The Active Case Ascertainment Method}

The active case ascertainment method has been employed in epidemiological studies of FASD conducted in the USA [11] and a number of other countries, including South Africa [32], Italy [38], and Croatia [39]. Unlike the surveillance method described above, the active case ascertainment approach has targeted all potential participants in a given population. For example, researchers have performed in-school screening evaluations of children attending a specific grade (e.g., grade one) in selected schools, using a multi-tier assessment approach. The multi-tier approach utilized by May et al. [40, 41] has involved three steps. First, all children in the targeted group are screened for growth restrictions (height and weight $<10$ th percentile) and physical signs of central nervous system dysfunction (head circumference $<10$ th percentile). In some studies, children with learning and behavioral problems have also been considered for further screening. Second, those who meet the growth criterion or the central nervous system dysfunction criterion undergo a morphological evaluation and a cognitive-behavioral assessment. Furthermore, maternal or informant caregiver interviews are conducted to gather information on alcohol exposure and other risk factors. Third, a decision regarding a diagnosis is made in a case conference after discussion of the morphological, neurobehavioral, and maternal data on a child. This methodology has allowed investigators to estimate the prevalence of FAS and partial FAS in a given community with a reasonable degree of accuracy. As shown in Table 1, investigators found high prevalence rates of FAS and partial FAS in certain communities in South Africa [12•, 32, 40, 42].

May et al. [12•] have recently estimated that the prevalence of FASD in certain communities in South Africa may be as high as $135.1-207.5$ per 1,000 . It is possible that lower rates reported for other countries reflect the moderating effects of sociocultural and economic factors. The main advantage of the active case ascertainment approach to identifying children with FASD is that it involves assessing most at risk children in a targeted group, thus allowing estimation of the prevalence with a reasonable degree of accuracy. By employing the multitier screening procedure, researchers have been successful in identifying previously unidentified children. For example, children identified through in-school screening in South Africa and Italy had not been previously diagnosed. A main limitation of this approach is that it is more expensive and time consuming than some other methods. Furthermore,

Table 1 Prevalence rates from in-school studies, listed by country

\begin{tabular}{|c|c|c|}
\hline & $\begin{array}{l}\text { FAS rate } \\
\text { per } 1,000\end{array}$ & $\begin{array}{l}\text { FASD rate } \\
\text { per } 1,000\end{array}$ \\
\hline $\begin{array}{l}\text { South Africa [44 May, 2000; } 47 \text { Viljoen, } \\
\text { 2005; } 46 \text { May, 2007; } 14 \text { May, 2013] }\end{array}$ & 50 & 72.3 \\
\hline $\begin{array}{l}\text { Washington, USA (13 Clarren, 2001) (Only } \\
\text { results obtained in one of the two screened } \\
\text { counties are shown, because of a low } \\
\text { consent rate in the second county) }\end{array}$ & 3.1 & \\
\hline US [Poitra, 2003] & $4.3^{*}$ & \\
\hline Italy, Europe [May, 2006; 15 May, 2011] & $4.0-8.2 *$ & $23.1-47.1 *$ \\
\hline US city 1 [May et al., 2009] & $1.4-2.5^{*}$ & $9.5-17.4^{*}$ \\
\hline US city 2 [May et al., 2009] & $6.4-11.3 *$ & $14.1-24.8^{*}$ \\
\hline $\begin{array}{l}\text { Plains Head Start School, USA [35 May } \\
\text { et al., 2009] }\end{array}$ & 10.2 & 20.3 \\
\hline Croatia, Europe [Petkovic and Barisic, 2010] & $16.9 *$ & $66.7^{*}$ \\
\hline Taiwan [Kuo et al., 2012] & $1.83^{*}$ & $5.76^{*}$ \\
\hline Korea [Lee, 2012] & $2.8^{*}$ & \\
\hline $\begin{array}{l}\text { General school } \\
\text { Institutions for intellectual disabilities }\end{array}$ & $14.9 \% *$ & \\
\hline $\begin{array}{l}\text { Croatia, Europe [Petkovic and Barisic, 2013] } \\
\text { Rural }\end{array}$ & $6.44 *$ & $40.77 *$ \\
\hline
\end{tabular}

\footnotetext{
* Percentages rated on sample-based estimate, not including children without parental consent. All of the other percentages are rated including children without parental consent (based on a denominator of the total number of children enrolled in the selected classrooms), hence assuming they were not affected
} 
because of the stigma attached to FASD, the parents of children with FASD are less likely to consent to participate in screening evaluations. Since most mothers are aware of the deleterious effects of alcohol in a developing fetus, they are likely to underreport drinking during pregnancy.

\section{Novel Methods to Determine Prenatal Alcohol Effects}

As Fig. 1 shows, two pieces of information are of critical importance in identifying children with prenatal alcohol exposure: confirmation that the child was exposed to alcohol, and confirmation that alcohol exposure has produced morphological alterations and/or functional deficits. As mentioned in the foregoing paragraphs, clinicians find it difficult to reliably obtain these pieces of information. It is known that maternal self-reports on the amount and frequency of alcohol intake during pregnancy could be inaccurate and that the stigma associated with fetal alcohol syndrome may lead even to denial of alcohol intake [13•]. To overcome this difficulty, researchers have turned to investigation of the utility of biomarkers of prenatal alcohol exposure. Similarly, as noted above, the clinical methods used to assess the effects of prenatal alcohol exposure in children have limitations. For example, only a small proportion of alcohol-exposed children display standard morphological alterations that are assessed in a clinical evaluation (growth restriction, microcephaly, malformations on the face). Therefore, researchers have begun to explore the utility of 3D facial imaging methods to look for minor anomalies that are not detectable by clinical morphological assessment. Furthermore, recent advances in neuroimaging methods have afforded an opportunity to answer the question as to whether children with prenatal alcohol exposure display a unique profile of structural and functional differences in the brain. In this section, we briefly review the latest findings made with these novel methodologies.

\section{Biomarkers of FASD}

The term "biomarkers" refers to cellular or molecular indices of exposure, disease, or susceptibility to disease [43]. As such, biomarkers have been classified into three broad categories: biomarkers of exposure, biomarkers of effects (disease), and biomarkers of susceptibility to disease [43]. Among the biomarkers of exposure or alcohol metabolism, fatty acid ethyl esters (FAEEs) have received considerable attention because they have been shown to correlate with moderate to heavy drinking during pregnancy [44]. Products of the nonoxidative metabolism of ethanol, FAEEs can be detected in the alcoholexposed infant's meconium, blood, cord blood, hair, and placenta. The sensitivity of FAEEs extracted from meconium has been found to vary widely [44-48], partly because of differences in study populations. Ostrea et al. [49] analyzed FAEEs in the meconium of 124 singleton infants and concluded that FAEEs in meconium, particularly ethyl linoleate and ethyl AA, are biomarkers of high specificity for exposure to alcohol prenatally. Peterson et al. [50] found that the presence of FAEEs in meconium is predictive of subsequent mental and psychomotor delays in children, but none of the children they examined had the dysmorphic features that characterize FAS or partial FAS. Therefore, the presence of FAEEs in meconium may help diagnose children with ARND. However, one difficulty with extracting FAEEs from meconium concerns the impracticality of collecting samples within a narrow window of time. Alcohol-induced alterations of body structure and function have been investigated as potential biomarkers of alcohol use. These include $\gamma$ glutamyltransferase (GGT), mean corpuscular volume (MCV), carbohydrate-deficient transferrin (CDT), dolichols, and proteomics [51, 52]. Although these biological markers are useful indicators of risky drinking, no single marker is diagnostically sensitive and specific enough to be considered a definitive biomarker of prenatal alcohol use [53]. More recently, investigators have begun exploring the possibility of extracting biomarkers from other sources such as newborn blood, hair, fingernails, placental tissue, and umbilical cord tissue. However, the usefulness of biomarkers extracted from cord blood, neonatal hair, and placental tissue depends on many factors [53]. For example, although amniotic fluid, cord blood, and placental tissue are easily available, as with meconium, the time window to collect them is narrow. Furthermore, while analyses of maternal or neonatal hair can indicate the timing of prenatal alcohol use, clinicians may not be able to collect them if the parents refuse or the infant has no hair. In general, despite a great deal of research to date, the goal of developing effective biomarkers that are able to definitively detect and quantify prenatal alcohol use remains elusive [10]. However, the use of biomarkers in conjunction with other procedures such as maternal self-report, morphological assessments, and neurocognitive/developmental assessments may aid the identification of alcohol-exposed children.

\section{D Facial Photography}

The standard morphological evaluation involves a physical examination aimed at the assessment of cardinal features typically associated with prenatal alcohol exposure. These include growth restrictions (weight and height $<10$ th percentile), evidence of atypical brain development (microcephaly), specific malformations on the face, and anomalies affecting other systems (skeletal, cardiac, etc.). A fundamental limitation of this morphological approach to identifying children with alcohol exposure is the absence of the foregoing anomalies in many alcohol-exposed children. Therefore, some investigators have sought to find subtle anomalies in alcohol- 
exposed individuals, using extended anthropometric protocols. Moore et al. [54, 55] investigated 21 craniofacial anthropometric measurements in 100 prenatally exposed individuals in an attempt find such subtle indicators of prenatal alcohol exposure. These investigators found that six anthropometric facial measurements discriminated alcohol-exposed individuals from controls [56]. Recently, novel 3D image analysis techniques have been developed with the aim of making more easily detectable the pattern of facial characteristics that are typical of FAS or partial FAS. 3D facial analysis using dense surface modeling (DSM) has proven successful initially in delineating facial morphology in Noonan syndrome, Williams syndrome, 22q11 deletion syndrome, Bardet-Biedl syndrome [57], and Smith-Magenis syndrome, and in discriminating controls from individuals with Noonan syndrome and 22q11 deletion syndrome $[17,58,59]$. The 3D facial laser scanner represents one of the most recent attempts at utilizing technology to characterize morphological anomalies associated with prenatal alcohol exposure [60]. In a recent international study of 149 individuals (86 with FAS and 63 controls), investigators utilized computer graphics, machine learning, and pattern recognition techniques to automatically identify a set of facial features that best discriminated individuals with FAS from controls in each sample. The aim of the study was to develop a computational model that can automatically compute facial features from $3 \mathrm{D}$ scans and use these data to accurately identify children with FAS. The results showed that computer algorithms could be used to automatically detect facial features that can discriminate between FAS and control faces. Thus, the 3D technologies have potential for detection of subtle features associated with prenatal alcohol exposure that are not detected in clinical evaluations [16]. However, a fundamental limitation of this technology is that it is expensive and so may not be clinically useful.

\section{Neuroimaging and Morphological/Functional Alterations in the Brain}

The last decade has witnessed tremendous advances in the application of modern neuroimaging methods to characterize structural and functional anomalies in children with prenatal alcohol exposure (for a comprehensive review, please see Riley et al. in this issue). Several structural magnetic resonance imaging (sMRI) studies have revealed a range of macrostructural anomalies in children and adolescents with prenatal alcohol exposure [61, 62], including reduction of overall brain volume [63] and reductions in the frontal, temporal, parietal, and - to a lesser degree - occipital lobes in children with FASD, relative to typically developing controls [63-65]. In studies using functional magnetic resonance imaging (fMRI), investigators have found significant differences in brain activation patterns during performance of various tasks [15]. A consistent pattern emerging from fMRI studies is that the FASD group activate neural structures different than those recruited by neurotypical controls during performance of cognitive tasks. For example, Sowell et al. [66] examined fMRI activation patterns of FASD and control groups during the performance of a verbal paired-associate learning task and observed distinct patterns of activation in the two groups. The FASD group was found to show less activation in the left medial and posterior temporal regions and more activation in the right dorsal frontal cortex. This pattern of results was interpreted as showing that the FASD group is compensating for deficient temporal lobe functions by recruiting the dorsolateral region. Using diffusion tensor imaging (DTI), investigators have obtained evidence of white matter abnormalities in FASD. A consistent finding emerging from DTI studies is that the FASD group show reduced white matter in many regions of the brain, particularly in the corpus callosum. For example, Wozniak et al. [67] have reported pronounced abnormalities in the corpus callosum, major anterior-posterior fiber bundles, corticospinal tracts, and cerebellum. Furthermore, Wozniak et al. [67] have found that anomalies in the white matter correlate with neurocognitive deficits, especially in processing speed, non-verbal ability, and executive functioning. Consistent with these findings are the results of magnetoencephalographic (MEG) studies reported by Stephen et al. [68••]. These investigators found delayed M100 responses to auditory and visual stimuli in the FASD group, in line with the slow information processing hypothesis in FASD [6].

\section{Conclusion}

Despite recent advances in the assessment of alcohol exposure and alcohol-induced brain damage, the identification of children with alcohol-related neurodevelopmental disorder (ARND) remains a challenging problem. There exist two main obstacles to identifying children with ARND in clinical practice. First, it remains challenging to obtain accurate data on prenatal alcohol exposure, because of inaccurate maternal reports or unavailability of mothers for interviews. In North America, many alcoholexposed children are placed in foster homes; therefore, accurate prenatal data are not often available. Second, children with FASD often have complex developmental histories notable for numerous adverse conditions that negatively impact brain development, such as exposure to violence, sexual and physical abuse, poverty, and malnutrition. Therefore, there is tremendous heterogeneity in the FASD group, making it hard to find a unique neurobehavioral profile or a biomarker profile associated with prenatal alcohol exposure. Despite these obstacles, there is 
hope that researchers will be able to develop new tools that can be employed to improve the identification of alcohol-exposed children clinically.

\section{Compliance with Ethics Guidelines}

Conflict of Interest Giovanna Coriale, Daniela Fiorentino, Piyadasa W. Kodituwakku, Luigi Tarani, Giovanni Parlapiano, Bruna Scalese, and Mauro Ceccanti declare that they have no conflict of interest.

Human and Animal Rights and Informed Consent This article does not contain any studies with human or animal subjects performed by any of the authors.

\section{References}

Papers of particular interest, published recently, have been highlighted as:

- Of importance

- Of major importance

1. Lemoine P, Harousseau H, Borteyru JP, Menuet JC. Children of alcoholic parents: abnormalities observed in 127 cases [in French]. Ouest Med. 1968;21:476-82.

2. Jones KL, Smith DW. Recognition of the fetal alcohol syndrome in early infancy. Lancet. 1973;2:999-1001.

3. Warren KT, Hewitt BG. Fetal alcohol spectrum disorders: when science, medicine, public policy, and laws collide. Dev Disabil Res Rev. 2009;15(3):170-5.

4. Stratton K, Howe C, Battaglia F. Fetal alcohol syndrome: diagnosis, epidemiology, prevention, and treatment. Washington, DC: National Academy Press; 1996.

5. O'Connor MJ, Blair P. Psychiatric conditions associated with prenatal alcohol exposure. Dev Disabil Res Rev. 2009;15(3):225-34.

6. Kodituwakku PW. Defining the behavioral phenotype in children with fetal alcohol spectrum disorders: a review. Neurosci Biobehav Rev. 2007;31:192-201.

7. Streissguth A. Offspring effects of prenatal alcohol exposure from birth to 25 years: the Seattle prospective longitudinal study. J Clin Psychol Med Settings. 2007;14:81-101.

8. Bearer $\mathrm{CF}$ et al. Biomarkers of alcohol use in pregnancy. Alcohol Res Health. 2004;28(1):38-43.

9. Pichini S, Marchei E, Vagnarelli F, Tarani L, Raimondi F, Maffucci $\mathrm{R}$, et al. Assessment of prenatal exposure to ethanol by meconium analysis: results of an Italian multicenter study. Alcohol Clin Exp Res. 2012;36(3):417-24.

10. Szabo G, Bakhireva LN, Savage DD. Focus on: biomarkers of fetal alcohol exposure and fetal alcohol effects. Alcohol Res Health. 2011;34(1):56-63.

11. Clarren SK, Randels SP, Sanderso M, et al. Screening for fetal alcohol syndrome in primary schools: a feasibility study. Teratology. 2001;63:3-10.

12. May PA, Blankenship J, Marais AS, Gossage JP, Kalberg W, Barnard R, et al. Approaching the prevalence of the full spectrum of fetal alcohol spectrum disorders in a South African population based study. Alcohol Clin Exp Res. 2013;37(5):819-30. This report provides data regarding prevalence of the full spectrum of FASD in a very high-risk population.

13. May PA, Fiorentino D, Coriale G, Kalberg WO, Hoyme HE, Aragón AS, et al. Prevalence of children with severe fetal alcohol spectrum disorders in communities near Rome, Italy: new estimated rates are higher than previous estimates. Int J Environ Res Public Health. 2011;8:2331-51. This is an interesting publication regarding the use of active case ascertainment in schools.

14. Spadoni AD et al. Neuroimaging and fetal alcohol spectrum disorders. Neurosci Biobehav Rev. 2007;31(2):239-45.

15. Astley SJ, Aylward EH, Olson HC, et al. Functional magnetic resonance imaging outcomes from a comprehensive magnetic resonance study of children with fetal alcohol spectrum disorders. J Neurodev Disord. 2009;1:61-80.

16. Wetherill L, Foroud T. Understanding the effects of prenatal alcohol exposure using three-dimensional facial imaging. Alcohol Res Health. 2011;34(1):38-41.

17. Hammond $P$ et al. Discriminating power of localized three-dimensional facial morphology. Am J Hum Genet. 2005;77:999-1010.

18. Sampson PD et al. Incidence of fetal alcohol syndrome and prevalence of alcohol-related neurodevelopmental disorder. Teratology. 1997;56(5):317-26.

19. Hymbaugh K, Miller LA, Druschel CM, Podvin DW, Meaney FJ, Boyle CA, et al. A multiple source methodology for the surveillance of fetal alcohol syndrome - the Fetal Alcohol Syndrome Surveillance Network (FASSNet). Teratology. 2002;66 Suppl 1:S41-9.

20. Centers for Disease Control and Prevention. Fetal alcohol syndrome-Alaska, Arizona, Colorado, and New York, 1995-1997. MMWR Morb Mortal Wkly Rep. 2002;51(20):433-5.

21. Centers for Disease Control and Prevention. Fetal alcohol syndrome-United States 1979-1992. MMWR. 1993;42:339-41.

22. Centers for Disease Control and Prevention. Update: trends in fetal alcohol syndrome-United States, 1979-1993. MMWR. 1995;44: 249-51.

23. Bower C, Silva D, Henderson TR, et al. Ascertainment of birth defects: the effect on completeness of adding a new source of data. J Paediatr Child Health. 2000;36:574-6.

24. Egeland GM, Perham-Hester KA, Gesaner BO, et al. Fetal alcohol syndrome in Alaska, 1977-1992: an administrative prevalence service from multiple sources. Am J Pub Health. 1998;88:781-6.

25. Clarren SK, Lutke J. Building clinical capacity for fetal alcohol spectrum disorder diagnoses in western and northern Canada. Can J Clin Pharmacol. 2008;15:e223-37.

26. Abel EL, Sokol RJ. Incidence of fetal alcohol syndrome and economic impact of FAS related anomalies. Drug Alcohol Depend. 1987;19:51-70.

27. Abel EL, Sokol RJ. A revised conservative estimate of the incidence of fetal alcohol syndrome and its economic impact. Alcohol Clin Exp Res. 1991;15:514-24.

28. Abel EL. An update on incidence of FAS: FAS is not an equal opportunity birth defect. Neurotoxicol Teratol. 1995;17:437-43.

29. Abel EL. Fetal alcohol abuse syndrome. New York: Plenum; 1998.

30. Abel EL. Fetal alcohol syndrome in families. Neurotoxicol Teratol. 1998;10:1-2.

31. May PA, Gossage JP, Kalberg WO, Robinson LK, Buckley DG, Manning M, et al. The prevalence and epidemiologic characteristics of FASD from various research methods with an emphasis on inschool studies. Dev Disabil Res Rev. 2009;15:176-92.

32. May PA, Gossage JP, Marais AS, et al. The epidemiology of fetal alcohol syndrome and partial FAS in a South African community. Drug Alcohol Depend. 2007;88:259.

33. May PA, Gossage JP, Marais AS, et al. Maternal risk factors for fetal alcohol syndrome and partial fetal alcohol syndrome in South Africa: a third study. Alcohol Clin Exp Res. 2008;32:738-53.

34. Kelly $\mathrm{Y}$ et al. Light drinking in pregnancy, a risk for behavioural problems and cognitive deficits at 3 years of age? Int J Epidemiol. 2009;38(1):129-40.

35. Kelly YJ et al. Light drinking during pregnancy: still no increased risk for socioemotional difficulties or cognitive deficits at 5 years of age? J Epidemiol Community Health. 2012;66(1):41-8. 
36. Sayal $\mathrm{K}$ et al. Light drinking in pregnancy and mid-childhood mental health and learning outcomes. Arch Dis Child. 2013;98(2):107-11.

37. Sood B et al. Prenatal alcohol exposure and childhood behavior at age 6 to 7 years: I. Dose-response effect. Pediatrics. 2001;108(2):E34.

38. May PA, Fiorentino D, Gossage JP, Kalberg W, Hoyme HE, Robinson LK, et al. Epidemiology of FASD in a province in Italy: prevalence and characteristics of children in a random sample of schools. Alcohol Clin Exp Res. 2006;30:1562-75.

39. Petkovic G, Barisic I. Prevalence of fetal alcohol syndrome and maternal characteristics in a sample of schoolchildren from a rural province of Croatia. Int J Environ Res Public Health. 2013;10:1547-61.

40. May PA, Brooke LE, Gossage JP, Croxford J, Adnams C, Jones $\mathrm{KL}$, et al. Epidemiology of fetal alcohol syndrome in a South African community in the Western Cape Province. Am J Public Health. 2000;90(12):1905-12.

41. Fiorentino D, Coriale G, Spagnuolo V, Prattero A, Attilia ML, Mancinelli R, et al. Fetal alcohol syndrome disorders: experience on the field. The Lazio Study preliminary report. Annali dell'Istituto Superiore di Sanità. 2006;42(1):56-63.

42. Viljoen DL, Gossage JP, Brooke L, et al. Fetal alcohol syndrome epidemiology in a South African community: a second study of a very high prevalence area. J Stud Alcohol. 2005;66:593-604.

43. Littner Y, Bearer CF. Detection of alcohol consumption during pregnancy - current and future biomarkers. Neurosci Biobehav Rev. 2007;31(2):261-9.

44. Bearer $\mathrm{CF}$ et al. Validation of a new biomarker of fetal exposure to alcohol. J Pediatr. 2003;143(4):463-9.

45. Bearer CF, Lee S, Salvator AE, et al. Ethyl linoleate in meconium: a biomarker for prenatal ethanol exposure. Alcohol Clin Exp Res. 1999;23(3):487-93.

46. Chan D, Baroz B, Pellerin B, et al. Population baseline of meconium fatty acid ethyl esters among infants of nondrinking women in Jerusalem and Toronto. Ther Drug Monit. 2003;25(3):271-8.

47. Derauf C, Katz AR, Easa D. Agreement between maternal selfreported ethanol intake and tobacco use during pregnancy and meconium assays for fatty acid ethyl esters and cotinine. Am J Epidemiol. 2003;158(7):705-9.

48. Moore C, Jones J, Lewis D, Buchi K. Prevalence of fatty acid ethyl esters in meconium specimens. Clin Chem. 2003;49(1):133-6.

49. Ostrea EM, Hernandez JD, Bielawski DM, et al. Fatty acid ethyl esters in meconium: are they biomarkers of fetal alcohol exposure and effect? Alcohol Clin Exp Res. 2006;30(7):1152-9.

50. Peterson J, Kirchner HL, Xue W, et al. Fatty acid ethyl esters in meconium are associated with poorer neurodevelopmental outcomes to two years of age. J Pediatr. 2008;152(6):788-92.

51. Halmesmaki E, Roine R, Salaspuro M. Gamma-glutamyl transferase, aspartate and alanine aminotransferases and their ratio, mean cell volume and urinary dolichol in pregnant alcohol abusers. Br J Obstet Gynaecol. 1992;99:278-91.

52. Wurst FM, Wiesbck GA, Metzger JW, Weinmann W. On sensitivity, specificity, and the influence of various parameters on ethyl glucuronide levels in urine: results from the WHO/ISBRA study. Alcohol Clin Exp Res. 2004;28(8):1220-8.

53. Bearer CF. Markers to detect drinking during pregnancy. Alcohol Res Health. 2001;25(3):210-8.

54. Moore ES et al. New perspectives on the face in fetal alcohol syndrome: what anthropometry tells us. Am J Med Genet. 2002;109(4):249-60.

55. Moore ES et al. Unique facial features distinguish fetal alcohol syndrome patients and controls in diverse ethnic populations. Alcohol Clin Exp Res. 2007;31(10):1707-13.

56. Moore ES et al. The subtle facial signs of prenatal exposure to alcohol: an anthropometric approach. J Pediatr. 2001;139(2):215-9.
57. Beales PL, Warner AM, Hitman GA, Thakker R, Flinter FA. Bardet-Biedl syndrome: a molecular and phenotypic study of 18 families. J Med Genet. 1997;34:92-8.

58. Hammond P, Hutton TJ, Allanson JA, Smith ACM. The 3D face of Smith-Magenis syndrome (SMS): a study using dense surface models. Eur J Hum Genet. 2003;11 Suppl 1:102-4.

59. Hammond P, Hindocha N, Hutton TJ, Beales PL. 3D dense surface modeling defines a characteristic facial phenotype in Bardet-Biedl syndrome. Am J Hum Genet. 2003;73 Suppl 1:284

60. Fang S, McLaughlin J, Fang J, Huang J, Autti-Rämö I, Fagerlund A, et al. Collaborative initiative on fetal alcohol spectrum disorders, automated diagnosis of fetal alcohol syndrome using 3D facial image analysis. Orthod Craniofac Res. 2008;11(3):162-71.

61. Lebel C, Rasmussen C, Wyper K, Walker L, Andrew G, Yager J, et al. Brain diffusion abnormalities in children with fetal alcohol spectrum disorder. Alcohol Clin Exp Res. 2008;32(10):173240.

62. Li L, Coles CD, Lynch ME, Hu X. Voxelwise and skeleton-based region of interest analysis of fetal alcohol syndrome and fetal alcohol spectrum disorders in young adults. Hum Brain Mapp. 2009;30(10):3265-74.

63. Chen X, Coles CD, Lynch ME, Hu X. Understanding specific effects of prenatal alcohol exposure on brain structure in young adults. Hum Brain Mapp. 2012;33:1663-76.

64. Archibald SL, Fennema-Notestine C, Gamst A, Riley EP, Mattson SN, Jernigan TL. Brain dysmorphology in individuals with severe prenatal alcohol exposure. Dev Med Child Neurol. 2001;43:148-54.

65. Sowell ER, Thompson PM, Mattson SN, Tessner KD, Jernigan TL, Riley EP, et al. Regional brain shape abnormalities persist into adolescence after heavy prenatal alcohol exposure. Cereb Cortex. 2002;12: $856-65$.

66. Sowell ER, Lu LH, O'Hare ED, McCourt ST, Mattson SN, O'Connor MJ, et al. Functional magnetic resonance imaging of verbal learning in children with heavy prenatal alcohol exposure. Neuroreport. 2007;18:636-9.

67. Wozniak JR, Bryon A, Mueller BA, Muetzel RL, Bell CJ, Hoecker $\mathrm{HL}$, et al. Inter-hemispheric functional connectivity disruption in children with prenatal alcohol exposure. Alcohol Clin Exp Res. 2011;35(5):849-61.

68.• Stephen JM, Kodituwakku PW, Kodituwakku EL, Romero L, Peters AM, Sharadamma NM, et al. Delays in auditory processing identified in preschool children with FASD. Alcohol Clin Exp Res. 2012;36(10): $1720-7$. This is an important publication regarding the use of auditory delay revealed by MEG as a neural marker of information-processing difficulties in young children with prenatal alcohol exposure.

\section{Table's References}

69. Poitra BA, Marion S, Dionne M, Wilkie E, Dauphinais P, Wilkie-Pepion M, et al. A school-based screening program for fetal alcohol syndrome. Neurotoxicol Teratol. 2003;25(6):725-9.

70. Petkovic G, Barisic I. FAS prevalence in a sample of urban schoolchildren in Croatia. Reprod Toxicol. 2010;29(2):237-41.

71. Kuo HT, Wu HP, Li Q, Zhou FC, Morisky DE, Lee ML. In school study of prevalence of fetal alcohol spectrum disorder in east Taiwan. Alcohol Clin Exp Res. 2012;36(Suppl S1):280.

72. Lee HK. Epidemiology of fetal alcohol syndrome in Korea: an active case ascertainment study. Alcohol Clin Exp Res. 2012;36(Suppl S1):212. 